\title{
Modos de integración lingüística en el alumnado de padres inmigrantes en España: un estudio de caso
}

\begin{abstract}
Alberto Álvarez-Sotomayor y M. Elena Gómez-Parra
En el análisis de la integración socioeducativa de los hijos de inmigrantes en España, la dimensión lingüística es una de las cuestiones de más interés. Pero existe un vacío empírico respecto a cómo la adquisición del español se conjuga con el mantenimiento o aprendizaje del idioma del país de origen. Este trabajo avanza en ese sentido. Mediante un estudio de caso sobre alumnado de secundaria, se analiza qué proporciones de hijos de inmigrantes se sitúan en cada uno de los cuatro modos de integración resultantes de dicha conjunción. Solo el $30 \%$ se encuadra dentro del bilingüismo competente, resultando la asimilación monolingüística el modo de integración más frecuente $(40 \%)$. Los resultados indican la necesidad de implementar nuevas estrategias institucionales para el fomento del multilingüismo en los hijos de inmigrantes.
\end{abstract}

Palabras claves: integración educativa; inmigración; estudiantes extranjeros; multilingüismo; España.

Types of language adaptation among immigrant students in Spain: a case study: Within the literature on social and educational integration of children of immigrants in Spain, the linguistic dimension has been one of the most observed. Nevertheless, the conjunction of the learning of Spanish and the learning or maintenance of the native language has scarcely been empirically analyzed. This paper moves into that direction. Through a case study on high school students, we analyze the distribution of children of immigrants along each of the four modes of integration resulting from such conjunction. Results show that only $30 \%$ of students in the analyzed subsample fall within the competent bilingualism type, and that monolingual assimilation is the most frequent type of integration $(40 \%)$. These results call for the need to implement new institutional strategies for the promotion of multilingualism among children of immigrants. 
Keywords: educational integration; immigration; foreign students; multilingualism; Spain.

\section{Introducción}

En el análisis de la integración socioeducativa de los hijos de inmigrantes en España, la integración lingüística es una de las cuestiones que más interés ha suscitado (García Castaño, Rubio y Bouachra 2015; Rodríguez Izquierdo 2009). En el ámbito político, la preocupación se ha centrado casi exclusivamente en el aprendizaje y el nivel adquirido en la lengua de la sociedad de llegada y vehicular de nuestro sistema educativo (a partir de aquí, y a efectos de simplificación, L2), el español en la mayoría de los casos. Así, desde un principio las medidas educativas más extendidas y a las que más esfuerzos han dedicado las comunidades autónomas son las relativas a la enseñanza de esa lengua vehicular (Terrén 2008).

En el ámbito académico, el interés por el aprendizaje de la lengua vehicular por parte de los hijos de inmigrantes está muy presente también (Martin et al. 2003; García Castaño et al. 2015). De hecho, son muchos los trabajos que han medido su dominio del español o de otras lenguas oficiales en España en el ámbito escolar, comparándolo con el de los hijos de nativos. La mayoría lo hacen a través de test lingüísticos y encuentran, por lo general, que los hijos de inmigrantes puntúan significativamente por debajo del alumnado autóctono en cuanto al conocimiento del español (Navarro, Huguet y Sansó 2014; González, Huguet y Chireac 2013; Oller y Vila 2008; Huguet, Navarro y Janés 2007; Mesa, Sánchez y Vázquez 1996) y del catalán en aquellas investigaciones desarrolladas en Cataluña (Navarro, Huguet y Sansó 2014; González, Huguet y Chireac 2013; Oller y Vila 2008; Vila 2008).

Pero, además, desde el ámbito académico español la preocupación respecto a la integración lingüística de este alumnado se ha centrado también en el mantenimiento de la lengua de su país de origen o del de los padres (L1) (García Castaño et al. 2015; Rodríguez Izquierdo 2009; Martin et al. 2003; Broeder y Mijares 2003). Este énfasis académico en el valor y la importancia de la enseñanza de la L1 se ha llevado a cabo, fundamentalmente, desde los preceptos de la educación intercultural. El argumento principal es que el aprendizaje de la L1 resulta clave para que estos jóvenes no pierdan su conexión con la cultura de origen, lo cual es condición sine qua non para que se produzcan situaciones de aprendizaje intercultural, donde existe diálogo, interacción e intercambio (Barrett 2013: 16). En este sentido, conviene distinguir el intercultural del resto de principales modelos de gestión educativa de la diversidad cultural.

Lengua y migración / Language and Migration 12:1 (2020), 77-99

Edición impresa: ISSN 1889-5425. Edición en línea: ISSN 2660-7166. (C) Universidad de Alcalá 
La asimilación-denominada anglo-conformity en los orígenes del término (Cole 1954)- describe la actitud de la cultura minoritaria por ajustarse a la cultura mayoritaria, que la acepta en tanto asuma sus principios y hábitos educativos. Por regla general, bajo este paradigma, la cultura mayoritaria ignora cualquier contribución de la cultura minoritaria, involucrándose esta en un proceso de adaptación (Abramitzky, Boustan y Eriksson 2017). La diferenciación aparece cuando se reconoce la coexistencia de diferentes grupos culturales pero no se reconoce la contribución de una cultura en la otra (Cortés y Dietz 2011). En relación a la educación, las contribuciones de la cultura minoritaria son reconocidas en el currículo escolar y, por tanto, la cultura mayoritaria ofrece diferentes posibilidades de escolarización a los niños de la cultura minoritaria. Un tercer modelo es el de la integración y aparece cuando la cultura mayoritaria reconoce las contribuciones de la cultura minoritaria en su haber, otorgándoles dicho estatus. El aprendizaje de la L2 es reconocido como un condicionamiento sine qua non para la integración del inmigrante (Polyankina 2012: 9; Vila 2000: 2), y los niveles de integración son mayores cuando los inmigrantes tienen intención de permanecer en el país de llegada que cuando piensan abandonarlo en un tiempo determinado (Huguet, Chireac, Navarro y Sansó 2011; Wachter y Fleischmann 2018). En el ámbito educativo, este modelo se produce cuando existe un tratamiento igualitario para todos los niños, sin que ninguno de ellos sea favorecido por su origen cultural (Porras 1998). El reconocimiento de los derechos culturales y educativos es completo, acogiendo el currículo escolar los aspectos más significativos de las minorías culturales. Finalmente, el modelo intercultural es aquel en el que las diferentes culturas contribuyen de igual a igual y en el que la planificación de la intervención educativa asume la diversidad cultural como una riqueza, asegurándose de que todos los miembros de todos los grupos culturales pueden beneficiarse de ella mediante la interacción y el intercambio mutuo (Schmelkes 2000). En este sentido, los centros escolares son considerados a menudo como un escenario ideal para la celebración de la diversidad y la educación intercultural (GómezHurtado, González-Falcón y Coronel 2016).

Sin embargo, pese a esta preocupación expresada desde posicionamientos interculturalistas por el mantenimiento de las "culturas de origen”, en España encontramos aún escasa la literatura empírica que mida la competencia lingüística de estos jóvenes en sus L1 y, lo que es más relevante a efectos de este trabajo, que mida simultáneamente la competencia en sus L1 y en la L2. En este terreno encontramos el trabajo de Broeder y Mijares (2003), que se vale de una encuesta con una amplia muestra $(n=24.429)$ realizada sobre el alumnado de primaria de Madrid en el curso 2000/01. En él analizan lo que denominan vitalidad de las 
lenguas de los alumnos basándose en su autopercepción. Dos de las dimensiones que componen este constructo son la "competencia lingüística" (a partir de la pregunta "¿qué lenguas habladas en casa puedes comprender, hablar, leer y escribir?”) y el "dominio lingüístico” (a partir de la pregunta “¿en qué lengua hablas mejor?”). Respecto a la primera, los resultados presentados muestran que la mayoría de los alumnos dicen comprender y hablar su lengua de origen, y en bastante menor medida leerla y escribirla. Las diferencias en función del grupo de origen son notables. Respecto a la segunda, los porcentajes de aquellos que afirman hablar esta lengua tan bien o mejor que el español oscilan fuertemente entre los distintos orígenes (desde solo el $14 \%$ entre los franceses hasta el $65 \%$ entre los chinos). Si bien estos datos suponen un importante antecedente, la no medición en forma de escala y el hecho de no presentar los datos de competencia lingüística respecto al español limitan el conocimiento de las pautas de integración lingüística del alumnado, objeto de estudio de nuestro trabajo. Así, por ejemplo, este estudio nos permite conocer qué porcentajes hablan la lengua de origen, pero no qué nivel tienen aquellos que lo hablan ni cómo es su nivel de español.

Más allá de las aportaciones del trabajo de Broeder y Mijares (2003), en España existe un importante vacío empírico a la hora de conocer la forma en la que el aprendizaje del español como L2 es conjugado por los hijos de inmigrantes con el mantenimiento o aprendizaje de su L1 y, por tanto, a la hora de conocer en qué medida las pautas de integración de estos jóvenes están encaminadas hacia determinadas formas de monolingüismo o de multilingüismo. El estudio simultáneo de su dominio en ambas lenguas -el cual permite definir el modo de integración lingüística de los inmigrantes- es de interés por, al menos, las siguientes razones. En primer lugar, porque el tipo de integración lingüística que lleven a cabo estos jóvenes condicionará su integración social, tanto en la sociedad de llegada, como en el seno de su grupo étnico. En segundo lugar, porque su modo de integración lingüística podrá afectar también a su integración educativa y socioeconómica en tanto en cuanto puede influir en su rendimiento académico (Bialystok 2011; Suárez-Orozco y Suárez-Orozco 2001) y en el capital humano que será tenido en cuenta cuando se incorporen al mercado laboral (Chiswick y Miller 2007). Y en tercer lugar, porque dicho análisis da pie a reflexionar acerca de la manera y la medida en las que las políticas y prácticas educativas lingüísticas implementadas en el contexto analizado pudieran estar contribuyendo al desarrollo de uno u otros modos de integración lingüística.

Tomando como modelo la tipología de Berry (1997) sobre las formas de aculturación, la cual, a efectos de simplificación, considera la existencia de dos ámbitos para la inclusión/exclusión de los individuos -sociedad de 
acogida y grupo étnico-, Esser (2006) distingue cuatro modos de integración lingüística (tabla 1): bilingüismo competente (cuando la persona tiene un grado de conocimiento y habilidad alto tanto en L1 en con L2), asimilación monolingüística (competencia elevada en L2 y baja en L1), segmentación monolingüística (nivel bajo en L2 y alto en L1), y marginalidad lingüística o bilingüismo limitado (nivel bajo en ambos idiomas). ${ }^{1}$ La marginalidad lingüística no promovería la inclusión ni en el grupo étnico ni en la sociedad de destino; la segmentación llevaría a la integración en el grupo étnico y a la exclusión en la sociedad de llegada; la asimilación lingüística -entendida en el sentido de aculturación-conduciría a la situación inversa a la anterior; mientras que el bilingüismo competente se sitúa en el escenario de la inclusión múltiple, es decir, en el de la inclusión en ambos sistemas sociales.

A diferencia de lo que ocurre en España, en el panorama internacional encontramos algunos referentes empíricos sobre esta cuestión. Según los datos de tres grandes encuestas realizadas en EE.UU. y Alemania (CILS, ${ }^{2}$ NELS y BMFSFJ), tres de las pocas con muestras amplias que ofrecen indicadores de competencia lingüística tanto en la lengua de origen como en la "nueva" lengua (Esser 2006: 49), la asimilación monolingüística sería la pauta predominante en los contextos analizados. En ella se encuadrarían en torno al $40 \%$ de cada una de las tres poblaciones encuestadas. Le seguiría el bilingüismo competente, con porcentajes que oscilan entre el $23 \%$ y el $31 \%$, y en menor medida, pero con porcentajes tampoco desdeñables, la segmentación monolingüística y el bilingüismo limitado.

\begin{tabular}{|c|c|c|c|}
\hline & \multicolumn{2}{|c|}{$\begin{array}{c}\text { Integración en sociedad de llegada/ } \\
\text { Competencia en L2 }\end{array}$} \\
\hline & & $\begin{array}{l}\text { Síl } \\
\text { Alta }\end{array}$ & $\begin{array}{l}\text { No/ } \\
\text { Baja }\end{array}$ \\
\hline \multirow{2}{*}{$\begin{array}{c}\text { Integración en } \\
\text { grupo étnico/ } \\
\text { Competencia } \\
\text { en LI }\end{array}$} & $\begin{array}{l}\text { Sí/ } \\
\text { Alta }\end{array}$ & $\begin{array}{c}\text { Inclusión múltiple/ } \\
\text { Bilingüismo competente } \\
\text { CILS........ 23\% } \\
\text { NELS...... 37\% } \\
\text { BMFSFJ... 31\% }\end{array}$ & $\begin{array}{c}\text { Segmentación/ } \\
\text { Segmentación monolingüística } \\
\text { CILS....... 16\% } \\
\text { NELS...... 16\% } \\
\text { BMFSFJ... 18\% }\end{array}$ \\
\hline & $\begin{array}{l}\text { No/ } \\
\text { Baja }\end{array}$ & $\begin{array}{c}\text { Asimilación/ } \\
\text { Asimilación monolingüística } \\
\text { CILS........ 40\% } \\
\text { NELS......39\% } \\
\text { BMFSFJ... 36\% }\end{array}$ & $\begin{array}{c}\text { Marginalidad/ } \\
\text { Bilingüismo limitado } \\
\text { CILS........ 21\% } \\
\text { NELS..... } 8 \% \\
\text { BMFSFJ... 15\% }\end{array}$ \\
\hline
\end{tabular}

Tabla 1. Modos de integración lingüística según la competencia en L1 y L2 Fuente: Esser (2006: 50). Elaboración propia 
Aplicando esta misma tipología, el presente trabajo tiene como objetivo principal describir y analizar cómo los estudiantes hijos de inmigrantes de un caso de estudio centrado en España conjugan la adquisición de la L2 (español) con el mantenimiento o el aprendizaje de sus L1 según lo reportado por ellos mismos en una encuesta. En la línea de trabajos anteriores, entendemos que el nivel lingǘstico auto-percibido refleja no solo su dominio, sino también su autoestima lingüística (Bourdieu 1991; Brizić 2006; Matias 2012). Se analiza para ello una muestra representativa de un caso de estudio centrado en el alumnado del último ciclo de Educación Secundaria Obligatoria (ESO) del municipio de Marbella, en la provincia de Málaga.

El trabajo queda estructurado del siguiente modo. Primero, se contextualiza el caso de estudio, prestando especial atención al marco político-administrativo en el que se encuadra. Ello permite conocer la regulación y las medidas que rigen las actuaciones relacionadas con la integración socioeducativa y lingüística de este alumnado. En segundo lugar, se especifican las cuestiones metodológicas más relevantes. Seguidamente se muestran los resultados de los análisis bi-variados, lo que supone describir cómo se distribuye la muestra empleada en los modos de integración lingüística señalados y cómo dicha distribución varía en función del tiempo de residencia en España. Se finaliza señalando las principales conclusiones del trabajo.

\section{Contexto político-administrativo del caso de estudio}

Desde finales de los años noventa hasta 2008 España vivió un boom inmigratorio que, en términos relativos, no tuvo parangón entre los países de la Unión Europea ni de la OCDE (Cebolla-Boado y GonzálezFerrer 2013: 36). Los nacidos fuera de España pasaron de representar el $2 \%$ al $16 \%$ del total de la población en edad de trabajar. Este cambio demográfico alcanzó también el sistema educativo. Así, según la estadística de enseñanzas no universitarias (Ministerio de Educación, Cultura y Deporte), el alumnado extranjero suponía en el curso 1997/98 un 1\% del total, mientras que en el curso 2008-09 representaba un 9,8\%. Dicho cambio llevó a que, en mayor o menor grado, las cuestiones vinculadas al binomio educación-inmigración entraran a formar parte de la agenda política de los gobiernos autonómicos.

A partir de entonces, en la mayor parte de comunidades autónomas comenzaron a diseñarse medidas que buscaban responder a esa nueva realidad. Pero, como corresponde por el reparto competencial existente

Lengua y migración / Language and Migration 12:1 (2020), 77-99

Edición impresa: ISSN 1889-5425. Edición en línea: ISSN 2660-7166. @ U Universidad de Alcalá 
en España, tales medidas han de ajustarse al marco general delimitado por las leyes estatales en materia educativa y de extranjería, respectivamente. A su vez, como Estado miembro de la UE, España sigue ciertas directrices no vinculantes marcadas por este organismo supranacional en cuestión de inmigración.

Por ello puede decirse que el marco concreto que rige la atención institucional al alumnado inmigrante en el caso que aquí se analiza está delimitado por las políticas y normativas desarrolladas en estos tres niveles político-administrativos: el comunitario (europeo), el nacional (español) y el autonómico (andaluz). En conjunción, y sintetizando, puede afirmarse que el marco en cuestión queda definido por los siguientes elementos clave (Álvarez de Sotomayor 2011):

1) El derecho a la educación de los extranjeros en las mismas condiciones que los españoles. Este queda recogido tanto en la Constitución española (art. 27), como en el artículo 9 de la Ley Orgánica 4/2000 sobre derechos y libertades de los extranjeros en España y su integración social.

2) Los principios de normalización e integración educativa. A partir de la Ley General de Ordenación del Sistema Educativo (LOGSE), de 1990, los alumnos y alumnas con «necesidades educativas especiales» reciben la enseñanza junto al resto de compañeros, teniendo todos como referencia un currículo común. Los inmigrantes quedan encuadrados en esta categoría por estar en situaciones sociales o culturales desfavorecidas, si bien también se incluirían en las referencias de esta y las sucesivas leyes educativas a las minorías étnicas, nacionales o culturales. Este principio se basa en la premisa de que la integración no solo repercute positivamente en las condiciones de aprendizaje de estos alumnos, sino que la diversidad que conlleva también beneficia al resto de alumnos y al conjunto del sistema.

3) El enfoque de la educación compensatoria. Con él, se plantean medidas que persiguen reducir el efecto de las desigualdades sociales en el ámbito educativo, procurando una mayor igualdad de oportunidades. La educación compensatoria se viene contemplando en España desde el Real Decreto 1174/1983, en el marco de la Ley General de Educación de 1970, pero es también a partir de la LOGSE y, sobre todo, del Real Decreto 299/1996, cuando se le otorga un mayor peso al promulgarse una mayor variedad de actuaciones y programas específicos. La LOGSE establece que "las políticas de educación compensatoria tienen como misión reforzar la acción del sistema educativo, de forma que se eviten las desigualdades derivadas de factores sociales, económicos, culturales, geográficos, étnicos o de otra indole“ (art. 63). Posteriormente, en el citado 
Decreto se especifica como uno de los tres grupos destinatarios prioritarios al alumnado perteneciente a minorías étnicas o culturales. En él, además, es donde por primera vez se hace referencia explícita al fenómeno de la inmigración.

4) El reconocimiento de la diversidad y la atención individualizada a la misma. También impulsado por la LOGSE y mantenido por las leyes sucesivas, la atención individualizada a la diversidad dentro del aula supone que el profesor ha de realizar ajustes para atender a las distintas capacidades, aptitudes y motivaciones del alumnado, y que se realizarán adaptaciones curriculares a los alumnos con necesidades especiales. Asimismo, y en línea con el enfoque intercultural, formalmente se establece un reconocimiento positivo de la diversidad de carácter cultural, étnico o nacional en el ámbito escolar y se propone el mantenimiento y la difusión de las culturas y lenguas propias, así como el impulso del desarrollo y el respeto de la identidad cultural del alumno (LOGSE, art. 10.2).

5) Medidas concretas de educación intercultural diseñadas por el Gobierno andaluz. En parte, promovidas desde la UE en el marco español ya descrito. Desde que a finales de los noventa se plantearan por primera vez políticas orientadas al alumnado de origen inmigrante en esta comunidad hasta 2007 (año de finalización del curso académico en el que se desarrolla esta investigación), todas las medidas diseñadas en esta materia en Andalucía quedan enmarcadas en los siguientes textos: la Ley de Solidaridad de la Educación de 1999, el Plan para la Atención del Alumnado Inmigrante en la Comunidad Autónoma Andaluza de 2001, y las secciones educativas del Primer y Segundo Plan Integral para la Población Inmigrante en Andalucía (PIPIA), de 2001 y 2006, respectivamente. Todos ellos -de contenido prácticamente idéntico entre sí- definen la orientación política de la comunidad autónoma andaluza en esta materia. Todos están en buena medida guiados por el «enfoque de la interculturalidad» -o al menos, se toma dicho concepto como uno de los ejes que lo vertebran-. En la adopción del mismo no hay que desestimar la influencia de la UE, que lleva impulsando dicho enfoque desde los años 80 a través, fundamentalmente, de disposiciones y recomendaciones del Consejo de Europa (Álvarez de Sotomayor 2011: 131).

En el seno de este marco, común en gran medida a todas las comunidades autónomas españolas, resulta comprensible que las medidas concretas destinadas a la integración educativa del alumnado de origen inmigrante se repitan bastante de unas a otras. Además, muchas de estas medidas han tenido como referencia las experiencias de otros países 
europeos. Terrén (2008) las distingue entre instrumentales, que tienen por destinatarios a este alumnado y a sus familias y que pretenden facilitar su incorporación y adaptación al sistema educativo; y expresivas, centradas más en valores, cuestiones culturales y de identidad. Entre ambas suelen cubrir tres líneas de actuación: acogida y mediación, compensación y apoyo, e identificación y sensibilización (ver tabla 2).

Según este esquema, en el ámbito lingüístico, las medidas destinadas al apoyo en el aprendizaje del español estarían encuadradas dentro de las instrumentales. Aquí situamos a las Aulas Temporales de Adaptación Lingüística (ATAL), que tienen como principal objetivo oficial "potenciar programas de apoyo para el aprendizaje de la lengua española" (Consejería de Educación y Ciencia 2001; Consejería de Gobernación 2001; 2006). En los análisis y valoraciones presentes en la literatura sobre esta clase de dispositivo - "aulas" de enseñanza-aprendizaje de la lengua vehicular- encontramos tanto trabajos que justifican y aprecian su existencia (Pomares y Pérez 2002; Bedmar 2002), como otros que se posicionan críticamente. Entre estos últimos, pueden distinguirse tres tipos de críticas (Terrén 2008): las de carácter político, que discuten la planificación, distribución y financiación de estos dispositivos; las que ponen en cuestión los supuestos sociolingüísticos en los que se basa su diseño; y las que consideran que en ellas se desarrollan prácticas asimilacionistas e incluso segregadoras que obstaculizan la integración de este alumnado.

\begin{tabular}{|c|c|c|c|}
\hline $\begin{array}{l}\text { Plano de } \\
\text { actuación }\end{array}$ & Población objeto & Objetivos & Tarea, medio \\
\hline \multirow[b]{3}{*}{ Instrumentales } & $\begin{array}{l}\text { Alumnado } \\
\text { inmigrante } \\
\text { y familia }\end{array}$ & Incorporación & $\begin{array}{l}\text { - Mediación, traducción, planes de } \\
\text { acogida, etc. }\end{array}$ \\
\hline & & Apoyo & - Inmersión lingüística (L2) \\
\hline & $\begin{array}{l}\text { Alumnado } \\
\text { inmigrante }\end{array}$ & $\begin{array}{l}\text { Desfases } \\
\text { escolares. } \\
\text { Compensación }\end{array}$ & $\begin{array}{l}\text { - Adaptaciones curriculares } \\
\text { - Refuerzo de asignaturas } \\
\text { - Otras medidas de compensación } \\
\text { educativa y de atención a la } \\
\text { diversidad }\end{array}$ \\
\hline \multirow[b]{2}{*}{ Expresivas } & $\begin{array}{l}\text { Alumnado } \\
\text { inmigrante }\end{array}$ & $\begin{array}{l}\text { Identidad de } \\
\text { origen }\end{array}$ & $\begin{array}{l}\text { Enseñanza de lengua y cultura de } \\
\text { origen }\end{array}$ \\
\hline & $\begin{array}{l}\text { Alumnado en } \\
\text { general }\end{array}$ & $\begin{array}{l}\text { Respeto y } \\
\text { conocimiento } \\
\text { intercultural. } \\
\text { Sensibilización }\end{array}$ & $\begin{array}{l}\text { - Jornadas y actividades } \\
\text { interculturales } \\
\text { - Campañas y acciones de } \\
\text { sensibilización } \\
\text { - Unidades didácticas }\end{array}$ \\
\hline
\end{tabular}

Tabla 2. Clasificación de medidas de integración educativa del alumnado inmigrante

Fuente: Terrén (2008) 
Valoraciones aparte, lo cierto es que, con diferentes denominaciones y modos de funcionamiento, este tipo de medida se ha puesto en marcha en la gran mayoría de las comunidades autónomas, siendo en todas ellas la que mayor esfuerzo ha acaparado por parte de las administraciones autonómicas (Rahona y Morales 2013). Así ocurre en Andalucía, donde las ATAL constituyen la iniciativa estrella no solo entre las instrumentales, sino del conjunto de medidas diseñadas en esta materia.

Por su parte, en el contexto español las iniciativas dedicadas a la enseñanza de la L1 se hallarían entre las medidas expresivas (Terrén 2008: 184). En el marco político-administrativo andaluz, estas quedan recogidas en el tercer objetivo del Plan para la Atención del Alumnado Inmigrante, "facilitar el aprendizaje de la lengua materna del alumnado para que éste no pierda la conexión con su cultura de origen" (Consejería de Educación y Ciencia 2001), y en el cuarto objetivo de los PIPIA I y II, "mantener y valorar la cultura de origen del alumnado" (Consejería de Gobernación 2001; 2006).

Andalucía es una de las ocho comunidades autónomas que ha llevado a cabo programas de aprendizaje y desarrollo de la lengua y cultura maternas (Rahona y Morales 2013: 65). Entre estos programas destacan por relevancia los ELCO (Enseñanza de la Lengua y la Cultura de Origen en grupos minoritarios). De hecho, en España el reconocimiento lingüístico de estas minorías se concibe casi únicamente en el marco de estos programas (Mijares 2006), ampliamente extendidos en el entorno europeo en la actualidad y que tienen sus primeros antecedentes en los años setenta en Francia, en el marco de las medidas dirigidas a los hijos de trabajadores europeos (Tilmatine, 1999). Los ELCO funcionan como acuerdos bilaterales en los que, en este caso, las autoridades españolas facilitan el acceso a los centros educativos, mientras que el país cuya lengua de origen quiere enseñarse provee el profesorado necesario a la vez que asume su remuneración. No obstante, diversos análisis han mostrado que este tipo de programas ha tenido un escaso nivel de implementación (Martínez de Lizarrondo 2009; Martín Rodríguez 2005; Mijares 2006; Ortiz Cobo 2005). Así, por ejemplo, en España únicamente se han establecido tres ELCO a nivel estatal, el marroquí -actualmente denominado LACM ${ }^{3}$ (Moscoso 2013)-, el portugués, y el rumano, y de ellos, en Andalucía solo se ha desarrollado el primero. Su baja implementación se comprueba a través del porcentaje de alumnado que ha accedido al programa. En el curso 2006-2007 -periodo analizado en este estudio de caso- participó en este programa solo el $6 \%$ del alumnado de origen marroquí matriculado en centros andaluces (Terrén 2008: 186).

Estos datos y análisis evaluativos ponen de manifiesto la existencia de una contradicción entre los modelos de integración lingüística presentes en el nivel legal y político-discursivo, por un lado, y en el nivel de 
la implementación y la práctica educativa, por otro. En el primero está claramente presente el enfoque intercultural, si bien, como ya se ha visto, podemos encontrarlo entremezclado con medidas más cercanas al asimilacionismo y al enfoque integracionista o compensador. Por el contrario, en la práctica -segundo nivel- la apuesta por la interculturalidad se desvanece. Lo hace en la medida en que la presencia de medidas orientadas a la enseñanza de las lenguas maternas de los hijos de inmigrantes es más bien testimonial -quedando su aprendizaje relegado al ámbito de lo privado-, mientras que la centralidad es ocupada por medidas que en un principio poseen un corte compensatorio y/o asimilacionista como las ATAL.

Es esta una tendencia no exclusiva de Andalucía, sino, de hecho, generalizada en el conjunto de España (Jiménez-Delgado 2015; Rodríguez Izquierdo 2009) y en la Unión Europea (Kraus 2008: 120). En este sentido, "desde el punto de vista lingüístico, los centros constituyen comunidades monolingües a pesar del multilingüismo de los estudiantes (...). Ni la Administración impulsa el mantenimiento y uso de las diferentes lenguas del alumnado, ni los profesores las incorporan a las prácticas educativas" (Rodríguez Izquierdo 2009: 12). Algunos estudios van más allá al afirmar que, al representarse al alumnado hijo de inmigrante como desaventajado tanto desde las administraciones educativas como desde el profesorado, se devalúan sus lenguas de origen y se refuerza la posición hegemónica de la lengua vehicular. De este modo, se reducirían, aplacarían o incluso invertirían las ventajas que tendría el bilingüismo o el potencial bilingüe de muchos de ellos (Martín 2010).

En el caso de Andalucía y del conjunto de España, esto contrasta fuertemente con la gran apuesta que ha supuesto el plurilingüismo respecto a la enseñanza instrumental de determinadas lenguas extranjeras -inglés, fundamentalmente-. Esta apuesta se visibiliza, por ejemplo, en el progresivo adelanto en la edad de comienzo de dicha enseñanza, que en la mayoría de regiones se establece ya en el segundo ciclo de Educación Infantil (de 3 a 6 años); en la adquisición de mayor peso curricular por parte de las asignaturas centradas en tal enseñanza; y en la implementación de múltiples medidas para la conversión de muchos centros en escuelas e institutos "bilingües", con otras asignaturas impartidas en esas lenguas además de las dedicadas específicamente a la instrucción de esos idiomas.

\section{Método y datos}

Para dar respuesta a las anteriores preguntas se explotan datos provenientes de la parte cuantitativa de un estudio de caso que tenía como objetivo principal analizar los factores que afectan al rendimiento aca- 
démico diferencial de los hijos de inmigrantes. El lingüístico era uno de los explorados.

Los datos proceden de una encuesta cumplimentada por los estudiantes durante una hora lectiva del último trimestre del curso 2006-07, y dirigida a todo el alumnado de $3^{\circ}$ y $4^{\circ}$ de ESO de los diez institutos públicos del municipio español de Marbella ( $\mathrm{N}=1.461)$. La encuesta realizada contempla el conjunto del universo a estudiar (todos los alumnos matriculados en el último ciclo de ESO en los centros públicos de Marbella). Los únicos alumnos que no completaron el cuestionario fueron los que no acudieron a clase durante la hora lectiva en la que la encuesta se llevó a cabo. Por tanto, en lugar de una muestra, contamos aquí con el censo de la población objeto de estudio, lo cual invalida la necesidad y el sentido de realizar inferencias estadísticas, siendo los resultados estadísticamente representativos del universo al que se circunscriben.

No obstante, dados los propósitos específicos de este trabajo, únicamente explotamos aquí los datos del alumnado con origen en países no hispanohablantes $(\mathrm{n}=231)$. No incluimos al de origen hispanohablante (176), puesto que en su caso no puede hablarse de integración lingüística en los términos planteados. De los treinta y dos orígenes nacionales que encontramos en esta sub-muestra, Marruecos (24,3\%), Francia (13\%), Alemania (11,7\%), Reino Unido (7,9\%), Ucrania (6,3\%), Brasil $(4,2 \%)$ y Filipinas $(3,8 \%)$ son los más representados.

La elección del municipio de Marbella como caso de estudio relevante dentro de la comunidad autónoma de Andalucía se basó en tres criterios apriorísticos (Álvarez-Sotomayor y Martínez-Cousinou 2016):

1. La existencia de una elevada proporción de alumnado extranjero matriculado en enseñanza secundaria, etapa educativa objeto de análisis. En Andalucía no hay datos disponibles sobre alumnado extranjero en el nivel municipal, pero contábamos con indicios claros de que su peso era importante en Marbella por medio de diversas fuentes. Así, según datos de la Consejería de Educación de la Junta de Andalucía, en la fecha del estudio Málaga (a la que pertenece Marbella) era la provincia andaluza con mayor volumen de alumnado extranjero y, según el Anuario Estadístico de Inmigración, Marbella era el segundo municipio andaluz en número total de extranjeros (Álvarez-Sotomayor y Martínez-Cousinou 2016).

2. El hecho de que dicho alumnado extranjero estuviera geográficamente concentrado en un número reducido de centros de secundaria -diez, en este caso-, de manera que se pudiese analizar optimizando el tiempo y los costes de la investigación.

3. La existencia de diversidad en el origen nacional de tales alumnos extranjeros. 
En cuanto a la etapa de enseñanza analizada (segundo ciclo de la ESO), su elección responde al hecho de que esta es la fase inmediatamente anterior al punto más crítico y definitorio de la que será la trayectoria educativa final del individuo, aquel en el que ha de optar entre no continuar los estudios para acceder directamente al mercado laboral, realizar algún módulo de formación profesional o cursar el bachillerato y tomar así el camino más orientado a la universidad.

Para medir el nivel del alumnado tanto en el español como en la/s len$\mathrm{gua} / \mathrm{s}$ de origen se crean indices de dominio lingüístico. Estos índices -uno por cada lengua sobre la que el estudiante afirme tener cierta competencia- se construyen a partir de las respuestas de los propios encuestados a una pregunta en la que se les pedía que valorasen en una escala de 1 a 5 (de "muy mal" a "muy bien") su dominio en los distintos idiomas respecto a las cuatro destrezas lingüísticas: comprensión oral, comprensión escrita (lectura), expresión oral (habla) y expresión escrita (escritura). El índice se define como la media aritmética de las valoraciones realizadas en estas cuatro dimensiones. El coeficiente alfa de Cronbach entre los cuatro ítems es 0,85 , lo que indica un alto nivel de consistencia interna. Aunque la forma óptima de medir esta cuestión es a través de una prueba objetiva y estandarizada, investigaciones lingüísticas previas han probado consistentemente la habilidad idiomática auto-percibida como un indicador fiable del conocimiento real que se posee de un idioma (Fishman 1969; Hakuta 1986; Stolzenberg y Tienda 1997). Ello ha llevado a que, entre otros, estudios de la magnitud del CILS (Portes y Rumbaut 2001) y el NELS (Mouw y Xie 1999) en EE.UU hayan incorporado tal indicador. De este último se adaptó la escala utilizada en nuestro cuestionario, la cual había sido validada previamente (Valdés y Figueroa 1994). Asimismo, la medición del nivel lingüístico autopercibido resulta de interés en la medida en que es también indicador de la autoestima lingüística (Bourdieu 1991; Brizić 2006; Matias 2012).

Conviene aclarar que aplicar la tipología de los modos de integración lingüística ya explicada implica clasificar dicotómicamente ("poseer un muy buen nivel" y "no poseer un muy buen nivel") los niveles de competencia en L1 y en L2 según los índices de dominio lingüístico correspondientes. Se ha considerado que el nivel de competencia lingüística es muy bueno cuando el valor en el índice es superior a 4,5.

\section{Resultados}

Aplicando la tipología desarrollada por Esser (2006) hallamos que el alumnado de la muestra se distribuye de la siguiente manera entre los cuatro modos de integración lingǘstica distinguidos. De cada diez 
alumnos originarios de países no hispanoparlantes, aproximadamente cuatro $(39,4 \%)$ responden a pautas que tienden a la asimilación monolingüística -lo que facilita su integración en la sociedad española y la dificulta en el plano del "grupo étnico"-, dos (20,3\%) se encuadrarían en la segmentación lingüística -con implicaciones inversas a las anteriores-, tres serían bilingües $(29,9 \%)$-lo que le abre las puertas en ambas direcciones-, y uno $(10,4 \%)$ se encuentra en situación de marginalidad lingüística -lo que obstaculiza su integración en cualquiera de los dos ámbitos- (ver tabla 3).

Estos resultados están en línea con lo hallado en los tres grandes estudios referenciados en páginas anteriores (ver tabla 1), que situaban a la asimilación monolingüística como el modo predominante, seguido del bilingüismo competente y con la segmentación monolingüística y la marginalidad lingüística ocupando los últimos lugares.

Desde la óptica del aprendizaje de la L2, eso supone que prácticamente el $70 \%$ de estos estudiantes alcanza un nivel muy elevado de español, mientras que el $30 \%$ restante aún percibiría que tiene algunos problemas con él. Desde la óptica del aprendizaje o mantenimiento de la L1, la mitad de los hijos de inmigrantes procedentes de países no hispanohablantes no tendría un nivel muy bueno en la lengua de origen de sus padres. Y desde la óptica conjunta del bilingüismo, solo el 30\% estaría en situación de bilingüismo competente. Es decir, solo tres de cada diez aprovechan plenamente el potencial bilingüe del escenario en el que viven: tener un padre y/o madre con lengua nativa distinta al español y estar expuestos continuamente y en multitud de contextos a este idioma por el hecho de vivir en España.

\begin{tabular}{|l|c|c|c|c|}
\hline $\begin{array}{c}\text { Destrezas } \\
\text { lingǘsticas }\end{array}$ & $\begin{array}{c}\text { Bilingüismo } \\
\text { competente }\end{array}$ & $\begin{array}{c}\text { Asimilación } \\
\text { monolingǘstica }\end{array}$ & $\begin{array}{c}\text { Segmentación } \\
\text { monolingüística }\end{array}$ & $\begin{array}{c}\text { Bilingüismo } \\
\text { limitado }\end{array}$ \\
\hline $\begin{array}{l}\text { Dominio en las 4 } \\
\text { destrezas }\end{array}$ & 29,9 & 39,4 & 20,3 & 10,4 \\
\hline $\begin{array}{l}\text { Escuchar } \\
\text { (comprensión } \\
\text { oral) }\end{array}$ & 35,6 & 28,3 & 27,0 & 9,0 \\
\hline Hablar & 29,6 & 33,0 & 27,0 & 10,3 \\
\hline Leer & 24,9 & 36,2 & 27,5 & 11,4 \\
\hline Escribir & 18,2 & 43,7 & 22,9 & 15,2 \\
\hline $\mathrm{N}$ & 69 & 91 & 47 & 24 \\
\hline
\end{tabular}

Tabla 3. Distribución porcentual de los inmigrantes de origen no hispanohablante según los modos de integración y las destrezas lingüisticas Fuente: Álvarez de Sotomayor (2011). Elaboración propia

Al distinguir los modos de integración en función de las cuatro destrezas (tabla 3), se observa que la tendencia al bilingüismo competente

Lengua y migración / Language and Migration 12:1 (2020), 77-99

Edición impresa: ISSN 1889-5425. Edición en línea: ISSN 2660-7166. ㄷ Universidad de Alcalá 
es mayor en las orales que en las escritas, y, dentro de cada uno de estos dos grupos, en las receptivas que en las productivas. Lo contrario ocurre con la tendencia a la asimilación monolingüística. Esto evidencia que la adquisición de las lenguas de origen es, en comparación con la del español, más débil en las destrezas que se adquieren de un modo más diferencial en la escuela, las más académicas (escritura y lectura).

A continuación se analiza la incidencia del tiempo de residencia. Antes de observar su efecto sobre la distribución entre los distintos modos de integración, conviene atender a su relación con el dominio lingüístico en las L1 y en la L2. Según el conocimiento acumulado, el tiempo de residencia en la sociedad de llegada correlaciona positivamente con el nivel en la lengua de dicha sociedad y negativamente con el nivel en la lengua de origen (Esser 2006). Los datos aquí explotados están en esa línea (ver tabla 4): a medida que es más larga la estancia en España, aumenta el dominio del español y disminuye el de la L1. A partir de los seis años de residencia, por un lado, la media en el dominio del español asciende al intervalo de lo que hemos definido como un nivel "muy alto" (superior a 4,5) y, por otro lado, desciende de ese nivel respecto al dominio de la L1.

\begin{tabular}{|l|c|c|c|c|c|c|c|c|c|c|c|c|c|c|}
\hline $\begin{array}{l}\text { Índice de } \\
\text { dominio } \\
\text { lingüístico }\end{array}$ & \multicolumn{2}{|c|}{$<2$ años } & \multicolumn{2}{|c|}{$2-3$ años } & \multicolumn{2}{|c|}{$4-5$ años } & \multicolumn{3}{|c|}{$6-9$ años } & \multicolumn{2}{|c|}{$\begin{array}{c}10 \text { o más } \\
\text { años }\end{array}$} & \multicolumn{2}{|c|}{$\begin{array}{c}\text { Nacidos en } \\
\text { España }\end{array}$} & \multicolumn{2}{|c|}{ Total } \\
\hline $\begin{array}{l}\text { "L2" } \\
\text { (español) }\end{array}$ & 3,61 & 0,62 & 3,94 & 0,53 & 4,31 & 0,59 & 4,56 & 0,53 & 4,55 & 0,47 & 4,81 & 0,37 & 4,55 & 0,58 \\
\hline $\begin{array}{l}\text { "LI" } \\
\text { (lengua de } \\
\text { origen) }\end{array}$ & 4,92 & 0,22 & 4,67 & 0,74 & 4,64 & 0,63 & 4,38 & 0,67 & 4,37 & 0,69 & 3,52 & 0,87 & 4,08 & 0,92 \\
\hline
\end{tabular}

Tabla 4. Dominio de L1 y L2 del alumnado de origen no hispanobablante según tiempo de residencia en España (media y desviación típica)

Fuente: Álvarez de Sotomayor (2011). Elaboración propia

Vista la relación existente entre el dominio lingüístico y el tiempo de residencia es fácil deducir que el efecto de esta última variable sobre el modo de integración lingüística en el que se encuadren los hijos de inmigrantes será igualmente relevante. Así, en la tabla 5 se observan las siguientes tendencias respecto a la primera generación (no nacidos en España). La asimilación solo se da entre quienes llevan más de cinco años de residencia -a medida que se va afianzando su conocimiento del español- y en ningún caso supone el escenario mayoritario. Paralelamente, a partir de ese periodo decrece fuertemente la segmentación, que es el modo mayoritario en el segmento de 0 a 5 años de estancia. Puede verse como preocupante el hecho de que, aun así, esta y el bilingüismo limitado -los modos más desfavorecedores en términos de 
integración en la sociedad de llegada- alcancen proporciones relativamente elevadas entre quienes llevan más de 5 años residiendo en España (en torno al $15 \%$ y al $20 \%$, respectivamente). En cuanto al bilingüismo competente, este es minoritario entre quienes llevan menos de 4 años y crece en los segmentos posteriores, convirtiéndose en el modo más frecuente entre quienes viven en este país desde hace más de 5 años.

Entre los hijos de inmigrantes nacidos en España (segunda generación), que suponen prácticamente el 50\% del alumnado analizado, las tendencias son muy distintas. A diferencia de lo observado entre la primera generación, entre estos, la segmentación monolingüística y el bilingüismo limitado son escenarios absolutamente minoritarios ( $2 \%$ y $7 \%$, respectivamente). La gran mayoría de estos jóvenes (70\%) se encuadra en el asimilacionismo monolingüístico, lo que también difiere sustancialmente de la tendencia entre la primera generación, para quienes este era un modo minoritario. Por último, solo una quinta parte $(21 \%)$ le sacaría partido a su potencial bilingüe.

\begin{tabular}{|c|c|c|c|c|c|c|c|}
\hline \multicolumn{2}{|c|}{$\begin{array}{c}\text { Tiempo de residencia en } \\
\text { España }\end{array}$} & \multirow{2}{*}{$\begin{array}{c}\text { Bilingüismo } \\
\text { competente }\end{array}$} & \multirow{2}{*}{$\begin{array}{c}\begin{array}{c}\text { Asimilación } \\
\text { monolingǘst. }\end{array} \\
0\end{array}$} & \multirow{2}{*}{$\begin{array}{c}\text { Segment. } \\
\text { monoling. }\end{array}$} & \multirow{2}{*}{$\begin{array}{c}\begin{array}{c}\text { Bilingüismo } \\
\text { limitado }\end{array} \\
0\end{array}$} & \multirow{2}{*}{$\begin{array}{c}\% \text { total } \\
100\end{array}$} & \multirow{3}{*}{$\begin{array}{l}\mathbf{N} \\
15\end{array}$} \\
\hline \multirow{2}{*}{ Menos de 2 años } & $\%$ filas & & & & & & \\
\hline & $\%$ columnas & 2,9 & 0 & 27,7 & 0 & 6,5 & \\
\hline \multirow{2}{*}{ De 2 a 3 años } & $\%$ filas & 25,0 & 0,0 & 65,0 & 10,0 & 100,0 & \multirow{2}{*}{20} \\
\hline & $\%$ columnas & 7,2 & 0 & 27,7 & 8,3 & 8,7 & \\
\hline \multirow{2}{*}{ De 4 a 5 años } & $\%$ filas & 44,4 & 0,0 & 50,0 & $5,6 \%$ & 100,0 & \multirow{2}{*}{18} \\
\hline & $\%$ columnas & 11,6 & 0 & 19,1 & 4,2 & 7,8 & \\
\hline \multirow{2}{*}{ De 6 a 9 años } & $\%$ filas & 50,0 & 15,4 & 15,4 & $19,2 \%$ & 100,0 & \multirow{2}{*}{26} \\
\hline & $\%$ columnas & 18,8 & 4,4 & 8,5 & 20,8 & 11,3 & \\
\hline \multirow{2}{*}{10 o más años } & $\%$ filas & 43,6 & 20,5 & 15,4 & $20,5 \%$ & 100,0 & \multirow[b]{2}{*}{39} \\
\hline & $\%$ columnas & 24,6 & 8,8 & 12,8 & 33,3 & 16,9 & \\
\hline \multirow{2}{*}{ Nacidos en Espña } & $\%$ filas & 21,2 & 69,9 & 1,8 & $7,1 \%$ & 100,0 & \multirow{2}{*}{113} \\
\hline & $\%$ columnas & 34,8 & 86,8 & 4,3 & 33,3 & 48,9 & \\
\hline $\mathrm{N}$ & & 69 & 91 & 47 & 24 & & 231 \\
\hline
\end{tabular}

Tabla 5. Modos de integración lingüística de los inmigrantes de origen no bispanobablante según el tiempo de residencia Fuente: Álvarez de Sotomayor (2011). Elaboración propia

Estos análisis en función del tiempo de residencia advierten de las posibles transiciones de unos modos de integración a otros. Entre otras cuestiones, esto implicaría que una gran parte de los que, según su propia evaluación, no tienen un dominio general muy bueno del español podrían encontrarse en esa posición transitoriamente. Así, y atendiendo ahora a los porcentajes por columnas, en la tabla 5 se aprecia que aquellos encuadrados en la segmentación lingüística llevaban en su mayoría 
relativamente poco tiempo viviendo en España (el 55\% tiene menos de cuatro años de residencia y el $75 \%$ menos de seis), mientras que los otros tres tipos de integración son más habituales entre los que cuentan con una estancia más dilatada. Por tanto, si han continuado residiendo en España es probable que una porción de esos jóvenes que afirmaban manejarse muy bien en la L1 pero que aún tenía problemas con la L2 haya acabado desplazándose hacia alguno de los otros tres modos. Exceptuando aquellos casos que se hayan podido encerrar en redes y comunidades co-étnicas fuertemente endogámicas -en el contexto analizado (Costa del Sol) la literatura da cuenta de algunas comunidades de este tipo entre británicos y alemanes (O’Reilly 2007)-, el desplazamiento más plausible es hacia uno de los dos que implican un alto nivel en la L2 (bilingüismo competente y asimilación monolingüística), ya que es altamente probable que su exposición al español haya sido muy alta y la edad en la que se encontraban no era muy tardía para la adquisición de una nueva lengua. Que haya sido el primero o el segundo dependerá, en gran parte, del grado de exposición tenido a las lenguas de origen en ámbitos no escolares (hogar, redes co-étnicas fuera del instituto,...), pues, como se ha visto, la enseñanza de estas en es el sistema educativo español es prácticamente inexistente.

Esta interpretación de los datos en función de la variable tiempo de residencia sugiere, por tanto, que todavía habría un amplio margen para el crecimiento del bilingüismo competente entre una población que, como ya se ha apuntado, se encuentra a priori en escenarios especialmente propicios para ello.

Por el contrario, los dos modos de integración que conllevan un nivel no muy alto en la L1 se dan casi exclusivamente entre quienes llevan más de cinco años residiendo en España. En estos casos, el movimiento hacia a otros modos al acumular tiempo de residencia en España se antoja más improbable por las mismas razones anteriormente dadas.

\section{Conclusiones}

Las cuestiones lingüísticas son una de las que más atención suelen recibir cuando en las sociedades de llegada se analiza la integración socioeducativa de los hijos de inmigrantes. Si bien la preocupación se centra fundamentalmente en el aprendizaje y competencia de estos en la lengua vehicular, cada vez adquiere más importancia analizar cómo y en qué medida dicho aprendizaje se combina con el mantenimiento o la adquisición de sus lenguas de origen. Importancia destacada, entre otras, por la perspectiva interculturalista, la cual ha cobrado presencia en las últimas décadas en Europa, tanto en el ámbito académico como en el polí- 
tico. En España, que desde finales de los noventa hasta 2008 experimentó el mayor crecimiento relativo en población extranjera entre los países de la OCDE, este interés apenas se ha traducido, no obstante, en trabajos que examinen empíricamente esa combinación lingüística. Este artículo se mueve en ese terreno.

Los datos aquí presentados ponen de manifiesto que, en el contexto analizado, la asimilación monolingüística es, de los cuatro modos de integración discernidos, el más frecuente. El bilingüismo competente -aquel que permite la inclusión en el grupo étnico y en la sociedad de destino, en el que muchos estudios encuentran un efecto diferencial positivo sobre el rendimiento académico, y modo predilecto desde los preceptos de la educación intercultural- está menos presente en la integración lingüística de los estudiantes hijos de inmigrantes. No obstante, cuando distinguimos entre primera y segunda generación observamos tendencias distintas: mientras en la primera el bilingüismo competente tiene más presencia que el asimilacionismo, en la segunda ocurre lo contrario; y mientras entre la segunda los modos que a priori conllevan una mayor problemática para la integración en la sociedad de llegada (segmentación monolingüística y bilingüismo limitado) tienen escasa presencia, entre la primera representan proporciones no desdeñables incluso después de largos periodos de permanencia.

Asimismo, la distinción según el tiempo de residencia en España advierte de las probables transiciones lingüísticas que ocurren de unos modos de integración a otros. Se pone de manifiesto que el mayor margen de crecimiento del bilingüismo competente se encuentra en la vía del mantenimiento de las L1. Igualmente, los análisis dejan entrever que la lectura y, sobre todo, la escritura en esas L1 son las destrezas lingüísticas en las que este alumnado percibe una adquisición más deficitaria cuando esta se compara con la del español.

Tal y como se ha analizado aquí y en estudios previos, en España las políticas de integración lingüística dirigidas a estudiantes extranjeros se han centrado casi exclusivamente en la enseñanza del español. Estos programas han concentrado los mayores esfuerzos por parte de las administraciones, mientras que las medidas de enseñanza de las lenguas de origen han tenido una presencia testimonial. La retórica y la práctica política en esta materia entran así en contradicción: mientras desde la primera se ha abrazado a menudo el interculturalismo, la segunda se situaba entre la educación compensatoria y el asimilacionismo. En contraste, los planes y medidas para la formación plurilingüe del alumnado respecto a la enseñanza instrumental de determinadas lenguas extranjeras -inglés, fundamentalmente- han conllevado un importante esfuerzo inversor por parte de las distintas administraciones educativas en los últimos años. Por tanto, en un contexto social marcado por el reconoci-

Lengua y migración / Language and Migration 12:1 (2020), 77-99

Edición impresa: ISSN 1889-5425. Edición en línea: ISSN 2660-7166. @ U Universidad de Alcalá 
miento de la importancia que para el individuo y para la sociedad tiene el plurilingüismo, y en un contexto educativo en el que tal reconocimiento se ha materializado en una notable dotación de peso académico y financiero hacia la enseñanza de las segundas lenguas insertas dentro del currículo académico, aquel plurilingüismo que es resultado del mantenimiento o aprendizaje de las L1 del alumnado de origen inmigrante ha quedado al margen del interés institucional en España y, en concreto, en Andalucía. Se infraexplota, así, el potencial plurilingüe de estos niños y jóvenes, tal y como, de hecho, se observa en los datos.

El desarrollo de estrategias encaminadas a la explotación de ese potencial (es decir, al aprendizaje o mantenimiento de las L1) ha quedado y queda relegado al espacio de la familia o de la "comunidad étnica", lo que supone un obstáculo. Además, en estos ámbitos es relativamente frecuente que estas estrategias se centren única o fundamentalmente en las competencias orales de la lengua, algo que también puede deducirse de nuestros datos. En sociedades modernas como la española, la familia y la comunidad étnica son ámbitos menos habituados a la alfabetización, la cual se efectúa fundamentalmente en las instituciones escolares. Esto ayuda a explicar el hecho de que el perfil "bilingüe" sea aún más minoritario en las competencias escritas y que en ellas gane peso la asimilación monolingüística. Es decir, esto ayuda a explicar el hecho de que en nuestros datos encontremos una proporción notable de alumnos/as que se consideran bilingües en cuanto a las destrezas orales, pero más bien monolingües (siendo dominante el español) en las escritas.

Entendemos que el conocimiento de esta realidad puede contribuir a la planificación de medidas o estrategias encaminadas a desarrollar en mayor medida el potencial multilingüe de este alumnado, sea desde el ámbito público o privado. Dicho esto, entendemos también que en España se hacen necesarios nuevos estudios que amplíen y profundicen en el conocimiento de esa realidad; estudios que cuenten con muestras de mayor tamaño y representativas de contextos más amplios, siendo esta la principal limitación del presente trabajo. Con ello se superaría también un segundo problema, el referido a la actualidad de los datos. No obstante, esta segunda limitación es relativa, pues entendemos que estos siguen siendo útiles para el avance en el conocimiento de esta cuestión habida cuenta de: (1) la ya comentada escasez de trabajos que exploten datos sobre el mismo objeto de estudio en España, tanto hasta el momento analizado como más recientemente; y (2) el hecho de que ni las medidas y procedimientos seguidos en el tratamiento de esta cuestión en la escuela, ni los orígenes de los flujos migratorios han variado sustancialmente durante este tiempo. 
Alberto Álvarez-Sotomayor Universidad de Córdoba

Departamento de Ciencias Sociales

y Humanidades.

Facultad de Ciencias de la Educación

Avda. San Alberto Magno s/n, 14071,

Córdoba, España.

aasotomayor@uco.es

ORCID: 0000-0001-6174-1740

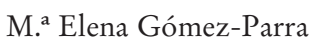

Universidad de Córdoba

Departamento de Filologías Inglesa y

Alemana

Facultad de Ciencias de la Educación

Avda. San Alberto Magno s/n, 14071

Córdoba, España.

elena.gomez@uco.es

ORCID: 0000-0001-7870-3505

\section{Notas}

1 Con diferente terminología, esta tipología es análoga a la usada en otros muchos trabajos (por ejemplo: Mouw y Xie 1999; Portes y Rumbaut 2001). Asimismo, el modelo de Berry (1997) ha sido y sigue siendo ampliamente empleado tanto en el ámbito internacional, en el que es un referente a pesar de que haya recibido críticas (Mesquita, De Leersnyder y Jasini 2017; Ward 2008), como en el español (Martin et al. 2003; Martín, Alcalá, Grad y Relaño 2005).

2 CILS (Children of Immigrants Longitudinal Study) se llevó a cabo en Florida y California desde 1991 hasta 2006; NELS (National Educational Longitudinal Study) en 1988, siendo representativa del conjunto de los EE.UU; y el BMFSFJ lo realizó en 2004 en Alemania el Ministerio Federal de familia, tercera edad, mujeres y juventud.

3 Programa de enseñanza de la Lengua Árabe y Cultura Marroquí.

\section{Referencias bibliográficas}

Abramitzky, Ran, Boustan, Leah y Eriksson, Katherine. 2017. "Cultural Assimilation during the Two Ages of Mass Migration”. Disponible en: http://www.economichistory.ca/pdfs/2017/abramitzky.pdf

Álvarez de Sotomayor, Alberto. 2011. El rendimiento académico de los alumnos inmigrantes en España: un estudio de caso. Tesis doctoral. Recuperado de http://0hera.ugr.es.adrastea.ugr.es/tesisugr/20152784.pdf (consultado 24 de septiembre de 2018).

Álvarez-Sotomayor, Alberto y Martínez-Cousinou, Gloria. 2016. “¿Capital económico o cultural? El efecto del origen social sobre las desventajas académicas de los hijos de inmigrantes en España”. Papers, 101:4. 527-554.

Barrett, Martin. 2013. "Interculturalism and multiculturalism: concepts and controversies". En Interculturalism and multiculturalism: similarities and differences, M. Barrett (ed.), 15-41. Strasbourg: Council of Europe.

Bedmar, Matías. 2002. "Educación social contra la exclusión: la interculturalidad". Estudios sobre las Culturas Contemporáneas, VIII:16. 83-95.

Berry, John W. 1997. Immigration, acculturation, and adaptation. Applied Psychology: An International Review, 46. 5-68.

Bialystok, Ellen. 2011. "Reshaping the Mind: The Benefits of Bilingualism". Canadian Journal of Experimental Psychology, 65:4. 229-35.

Bourdieu, Pierre. 1991. Language and symbolic power. Cambridge: Polity Press.

Brizic, Katharina. 2006. "The secret life of languages. Origin-specific differences in L1/L2 acquisition by immigrant children". International Journal of Applied Linguistics, 16:3. 339-362.

Cebolla Boado, Héctor y González Ferrer, Amparo. 2013. Inmigración: ¿integración sin modelo?. Madrid: Alianza Editorial.

Lengua y migración / Language and Migration 12:1 (2020), 77-99

Edición impresa: ISSN 1889-5425. Edición en línea: ISSN 2660-7166. (C) Universidad de Alcalá 
Chiswick, Barry R. y Miller, Paul W. 2007. The economics of language: International analyses. New York: Routledge.

Cole, Stewart G. 1954. Minorities and the American promise: The conflict of principle and practice. New York: Harper.

Consejería de Educación y Ciencia de la Junta de Andalucía. 2001. Plan para la atención educativa del alumnado inmigrante en la Comunidad Autónoma Andaluza. Sevilla: Signatura Ediciones.

Consejería de Gobernación de la Junta de Andalucía. 2001. Primer Plan Integral para la Inmigración en Andalucía. Sevilla: Consejería de Gobernación.

Consejería de Gobernación de la Junta de Andalucía. 2006. Segundo Plan Integral para la Inmigración en Andalucía. Sevilla: Consejería de Gobernación.

Cortés, Laura Selene Mateos y Dietz, Gunther. 2011. Interculturalidad y educación intercultural en México: Un análisis de los discursos nacionales e internacionales en su impacto en los modelos educativos mexicanos (en línea). Disponible en: http://www.interculturalidadygenero-colsan.com.mx/v4/pdf/GUNTHER\%20DIETZ.pdf (consultado 25 de septiembre de 2018).

Esser, Hartmut. 2006. Migration, Language and Integration. AKI Research. Review 4. Berlin: Wiss Berlin Sozial (WZB) (en línea). Disponible en: https://wzb.eu/www2000/alt/aki/files/ aki_research_review_4.pdf (consultado 03 de marzo de 2018).

Fishman, Joshua A. 1969. "The Validity of Census Data on Bilingualism in a Puerto Rican Neighborhood”. American Sociological Review, 34. 636-50.

García Castaño, Francisco Javier, Rubio Gómez, María y Bouachra, Ouafaa. 2015. "Immigrant Students at School in Spain: Constructing a Subject of Study". Dve domovini/Two Homelands, 41. 35-47.

Gómez-Hurtado, Inmaculada, González-Falcón, Inmaculada y Coronel, José M. 2016. "Perceptions of secondary school principals on management of cultural diversity in Spain. The challenge of educational leadership". Educational Management Administration E Leadership. 1-16. DOI: https://doi.org/10.1177/1741143216670651.

González Riaño, Xosé, Huguet, Ángel y Chireac, Silvia. 2013. “Diversidad lingüística y conocimiento de catalán/castellano. Un estudio empírico con alumnado inmigrante en Cataluña”. Teoría de la Educación, 25:1. 191-213.

Hakuta, Kenji. 1986. Mirror of Language: The Debate on Bilingualism. New York: Basic Books.

Huguet, Ángel, Chireac, Silvia-María, Navarro, José-Luis y Sansó, Clara. 2011. Tiempo de estancia y aprendizajes lingüísticos. El caso de los escolares inmigrantes en Cataluña. Cultura y Educación, 23:3. 355-370. DOI: 10.1174/113564011797330252.

Huguet, Ángel., Navarro, J. Luis y Janés, Judith. 2007. “La adquisición del castellano por los escolares inmigrantes. El papel del tiempo de estancia y la lengua familiar”. Anuario de Psicología, 38:3. 357-375.

Jiménez-Delgado, María. 2016. "Discursos interculturales y prácticas asimilacionistas: algunas contradicciones en el sistema educativo español”. Convergencia, 23:71. 41-61.

Kraus, Peter A. 2008. A union of diversity: language, identity and policy-building in Europe. New York: Cambridge University Press.

Kuncel, Nathan R., Credé, Marcus y Thomas, Lisa. 2005. “The validity of self-reported grade point averages, class ranks, and test scores: a meta-analysis and review of the literature". Review of Educational Research, 75:1. 63-82.

Martínez de Lizarrondo, Antidio. 2009. "Políticas autonómicas de integración de inmigrantes: la educación”. Revista Española de Educación Comparada, 15. 251-276.

Martín Rodríguez, José. 2005. La atención educativa del alumnado inmigrante: la provincia de Granada. Tesis doctoral inédita. Granada: Universidad de Granada.

Martín Rojo, Luisa. 2010. Constructing Inequality in Multilingual Classroom. Berlin, Germany: De Gruyter Mouton.

Martín Rojo, Luisa, Alcalá Recuerda, Esther, Gari Pérez, Aitana, Mijares, Laura, Sierra Rodrigo, Inmaculada y Rodríguez, M. Ángeles. 2003. ¿Asimilar o integrar?: dilemas ante el multilingüismo en las aulas. Madrid: Ministerio de Educación. 
Martín Rojo, Luisa, Alcalá Recuerda, Esther, Grad, Héctor y Relaño Pastor, A. María. 2008. "Lengua, identidad nacional y relaciones interculturales en la escuela". Puntos de Vista: Cuadernos del Observatorio de las Migraciones y la Convivencia Intercultural de la Ciudad de Madrid, 14. 27-47.

Martin, Marilyn Jones. 2007. "Bilingualism, Education and the Regulation of Access to Language Resources”. En Bilingualism: A Social Approach, M. Heller (ed.), 161-183. Hampshire: Palgrave Macmillan.

Matias, Ana Raquel Monteiro. 2012. Self-reported bilingual outcomes and language acculturation among descendants of Turkish immigrants in France, Germany and the Netherlands. Tesis doctoral inédita (en línea). Disponible en: https://repositorioiul.iscte.pt/handle/10071/7658 (consultado 03 de marzo de 2018).

Mesa, Ma Carmen, Sánchez, Sebastián y Vázquez, Ma del Mar. 1996. Educación y situaciones bilingües en contextos multiculturales. Estudio de un caso: Melilla. Granada: Laboratorio de Estudios Interculturales-CIDE.

Mesquita, Batja, De Leersnyder, Jozefien y Jasini, Alba. (2017). "The cultural psychology of acculturation". En Handbook of cultural Psychology, S. Kitayama y D. Cohen (ed.). New York: Guildford Press.

Mijares Molina, Laura. 2006. Aprendiendo a ser marroquíes: inmigración y escuela en España. Madrid: Ediciones del Oriente y del Mediterráneo

Moscoso García, Francisco. 2013. “El programa hispano-marroquí de enseñanza de Lengua Árabe y Cultura Marroquí (LACM) sometido a revisión. Árabe marroquí y amazige, lenguas nativas (L1)”. Anaquel de Estudios Árabes, 24. 119-135.

Mouw, Ted y Xie, Yu. 1999. "Bilingualism and the academic achievement of first- and second-generation Asian Americans: accommodation with or without assimilation?”. American Sociological Review, 64:2. 231-252.

Navarro, J. Luís, Huguet, Ángel y Sansó, Clara. 2014. “Competencias lingüísticas y alumnado inmigrante en Cataluña. El caso del colectivo de origen hispanohablante”. Educación XX1, 17(2): 361-382.

Oller, Judith. y Vila, Ignasi. 2008. “El conocimiento del catalán y el castellano del alumnado de origen extranjero, tiempo de estancia en Cataluña y lengua inicial al finalizar la enseñanza primaria”. Segundas lenguas e inmigración en red, I:1. 10-24.

O’Reilly, Karen. 2007. “Intra-European Migration and the Mobility-Enclosure Dialectic”. Sociology, 41:2. 277-293.

Ortiz Cobo, Mónica. 2005. Alumnado extranjero del sistema educativo andaluz. Racialismo en el discurso y práctica escolar. Tesis doctoral. Granada: Universidad de Granada.

Polyankina, Sonia Yu. 2012. Integration and Differentiation in Education in the Epoch of Globalization. Antologías de Conferencias de Ciencias Sociales, 16. 7-10.

Pomares Grancha, Jerónimo y Pérez López, José Manuel. 2002. "El ATAL: camino hacia la interculturalidad”. Organización y Gestión Educativa: Revista del Fórum Europeo de Administradores de la Educación, 10:2. 27-29. Disponible en: http://dialnet.unirioja.es/ servlet/articulo?codigo=238326 (consultado 03 de marzo de 2018).

Porras Vallejo, Ramón. 1998. Una escuela para la integración educativa. Una alternativa al modelo tradicional. Sevilla: M.C.E.P.

Portes, Alejandro y Rumbaut, Rubén G. 2001. Legacies: The Story of the Immigrant Second Generation. Los Angeles: University of California Press.

Rahona López, Marta y Morales, Serafín. 2013. Educación e inmigración en España: desafíos y oportunidades. Madrid: Organización de Estados Iberoamericanos.

Rodríguez Izquierdo, Rosa M. 2009. “The Research on Intercultural Education in Spain”. Education Policy Analysis Archives, 17. 1-29. Disponible en: http://www.scopus.com/ inward/record.url? eid=2-s2.0-62649151356\&partnerID=40\&md5=fac34950700b7342f9 bb937645468b63 (consultado 03 de marzo de 2018).

Schmelkes, Sylvia. 2000. La educación intercultural: un campo en proceso de consolidación. Ciudad de Méjico: Red Revista Mexicana de Investigación Educativa.

Lengua y migración / Language and Migration 12:1 (2020), 77-99

Edición impresa: ISSN 1889-5425. Edición en línea: ISSN 2660-7166. C C Universidad de Alcalá 
Stolzenberg, Ross M. y Tienda, Marta. 1997. "English Proficiency, Education, and the Conditional Economic Assimilation of Hispanic and Asian Origin Men”. Social Science Research, 25. 26-51

Suárez-Orozco, Carola y Suárez-Orozco, Marcelo. 2001. Children of Immigration. Cambridge: Harvard University Press.

Terrén Lalana, Eduardo. 2008. La integración educativa de los hijos de familias inmigradas. Documento de trabajo para el VI Informe FOESSA (en línea). Disponible en: http://www.foessa.es/publicaciones_compra.aspx?Id=38698Idioma=1\&Diocesis=42.

Tilmatine, Mohand. 1999. "La importancia de la lengua y cultura de origen en la integración de las comunidades bereberes en Europa”. En Lengua y cultura de origen: niños marroquies en la escuela española, A. Franzé (ed.), 143-156. Madrid: Ediciones del Oriente y del Mediterráneo.

Valdés, Guadalupe y Figueroa, Richard A. 1994. Bilingualism and Testing: A Special Case of Bias. Norwood: Ablex.

Vila, Ignasi. 2008. "Lengua familiar y conocimiento de la lengua escolar en Cataluña al finalizar la Educación Infantil”. Revista de Educación, 346: 401-424.

Wachter, Gusta A. y Fleischmann, Fenella. 2018. "Settlement Intentions and Immigrant Integration: The Case of Recently Arrived EU-Immigrants in the Netherlands". International Migration. Disponible en: https://onlinelibrary.wiley.com/doi/ full/10.1111/imig.12434 (consultado el 03 de abril de 2019).

Ward, Colleen. 2008. “Thinking outside the Berry boxes: New perspectives on identity, acculturation and intercultural relations”. International Journal of Intercultural Relations, 32:2. 105-114. 